\title{
Discrimination of blasts in mine seismology
}

\author{
D. Malovichko Institute of Mine Seismology, Australia
}

\section{Abstract}

Seismic monitoring systems installed in mines record seismic signals from various dynamic processes fracturing in rock mass, production and development blasts, impacts and vibration of machinery, etc. It is important to classify the recorded seismic events before doing most kinds of analysis of seismic data. For instance, the assessment of seismic hazard or analysis of rock mass stability requires only events associated with fracturing in rock mass.

A discrimination technique developed in global seismology was adopted for mining environments in order to identify blasts. The technique utilises a set of seismic event characteristics (time of occurrence relative to blasting time, radiation pattern, distribution of seismic energy between low-and high-frequency bands, correlation of the seismic signals with the preceding and succeeding waveforms) and quantifies a probability that a particular event belongs to a population of blasts.

The application of the technique to several mines is discussed.

\section{Introduction}

There is a variety of dynamic processes in mines which radiate seismic waves that can be detected by seismic monitoring systems. In general two types of processes play a key role (Figure 1). The first process is a development or production blast, when a rapid chemical reaction of explosive material causes crushing and fracturing in the surrounding rock mass. This initiates a disturbance that propagates outwards, predominantly in a form of a compressional seismic wave. The second process represents a shear fracturing or a sudden slip on a surface of weakness that generates vibrations in the surrounding rock mass. The corresponding seismic events will be referred to as a 'blast' for the first process, and a 'normal event' for the second process.

Standard interpretation and analysis of seismic monitoring data require these events to be separated. For instance, the analysis of rock mass stability (Mendecki, 1997) utilises normal events only. Presence of blasts in the seismic catalogue may result in the wrong interpretation, e.g. fictitious regions of high seismic stress.

The usual practice of processing seismic data includes a qualitative or semi-quantitative classification of seismic events. A person that processes the data decides whether an event is a normal event or a blast, based on features of the seismic waveforms (repetition of the signals, ratio of amplitudes of P- and S-waves) or source parameters (ratio of seismic energies estimated from P- and S-waves, time of day). This approach lacks consistency, since two processors may have different opinions regarding the features of the same event. One approach to eliminate blasts from the seismic catalogue is to apply time and/or spatial filters, i.e. events located close to blasting areas and/or at the blasting time are marked as blasts. This approach cannot be considered as universally applicable since it is possible to have a scenario where a blast triggers shear fracturing that radiates stronger seismic signals than the blast itself. In this case, the recorded event that occurs during the blasting time and locates close to the blast origin, has to be classified as 'normal'. 

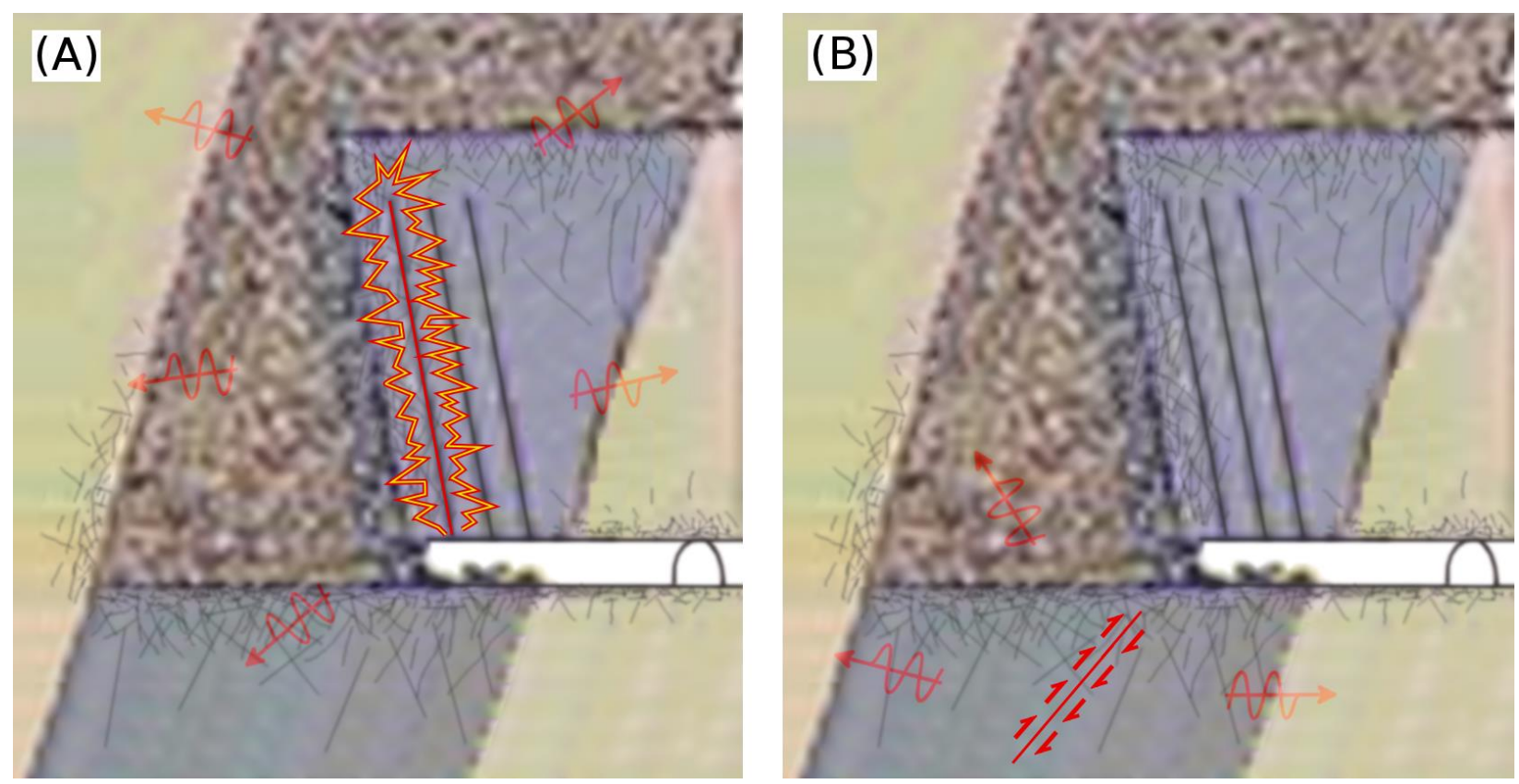

Figure 1 Two typical seismic source processes in mines - blast (A) and shear fracturing (B)

A similar problem of classifying seismic events exists in global seismology. A lot of research was done to develop algorithms that allow one to discriminate nuclear explosions from natural (tectonic) earthquakes. Various characteristics of seismic signals or seismic sources were found to be useful for this task, for instance:

- the ratio of amplitudes of P- and S-waves (in various frequency ranges)

- the ratio of amplitudes of body and surface waves

- depth of the source

- the source mechanism.

Usually the discrimination is based on a combination of these features. A popular discrimination technique is a multivariate maximum-likelihood Gaussian classifier (Booker and Mitronovas, 1964; Taylor, 1996).

This paper describes the application of this technique to the mining environments.

\section{Method}

\section{1 Theory}

The characteristics of waveforms or seismic sources that differ for blasts and normal events are called discriminating features. The selected set of features composes a vector $\mathbf{V}$. It is assumed that the population of blasts, $\mathbf{X}$, have features $\mathbf{v}$ belonging to a multivariate Gaussian distribution with mean $\boldsymbol{\mu}_{\mathbf{X}}$ and covariance matrix $\boldsymbol{\Sigma}_{\mathbf{X}}$, while the features of the normal event population, $\mathbf{Q}$, belong to a Gaussian distribution with mean $\boldsymbol{\mu}_{\mathrm{Q}}$ and covariance matrix $\boldsymbol{\Sigma}_{\mathrm{Q}}$.

The discrimination function:

$$
G(\mathbf{v})=\left[-\frac{1}{2}\left(\mathbf{v}-\boldsymbol{\mu}_{\mathbf{X}}\right)^{T} \boldsymbol{\Sigma}_{\mathbf{x}}^{-1}\left(\mathbf{v}-\boldsymbol{\mu}_{\mathbf{X}}\right)\right]-\left[-\frac{1}{2}\left(\mathbf{v}-\boldsymbol{\mu}_{\mathbf{Q}}\right)^{T} \boldsymbol{\Sigma}_{\mathbf{Q}}{ }^{-1}\left(\mathbf{v}-\boldsymbol{\mu}_{\mathbf{Q}}\right)\right]+\frac{1}{2} \ln \frac{\left|\boldsymbol{\Sigma}_{\mathbf{Q}}\right|}{\left|\boldsymbol{\Sigma}_{\mathbf{X}}\right|}
$$


is used to separate the populations of blasts and normal events.

If $G(\mathbf{v})>0$ the event is classified as a blast, while in the opposite case $(G(\mathbf{v}) \leq 0)$, it is classified as a normal event.

Making use of the technique consists of two phases:

- Calibration - representative sets of blasts and normal events are manually selected to derive vectors $\boldsymbol{\mu}_{\mathrm{x}}$ and $\boldsymbol{\mu}_{\mathrm{Q}}$, and matrices $\boldsymbol{\Sigma}_{\mathrm{x}}$ and $\boldsymbol{\Sigma}_{\mathrm{Q}}$.

- Application - for an event of interest, the features $\mathbf{v}$ are calculated and expression (1) is evaluated.

\section{2 Discriminating features}

The discriminator performs better when the discriminating features are physically independent of each other, i.e. they characterise different aspects of the sources of blasts and normal events. Due to this the following features were selected:

- time of day

- repetition of the waveforms

- high-frequency versus low-frequency radiation

- radiation pattern.

\subsection{Time of day}

Generally, mines have prescribed blasting times. The probability of an event being a blast is higher during these times. The 'time of day' feature measures the difference between the event's time and the closest blasting time. This feature was found to be efficient in several mines.

\subsection{Repetition of the waveforms}

Blasts are frequently characterised by a repetition of similar signals within a short time interval. An example is shown in Figure 2.

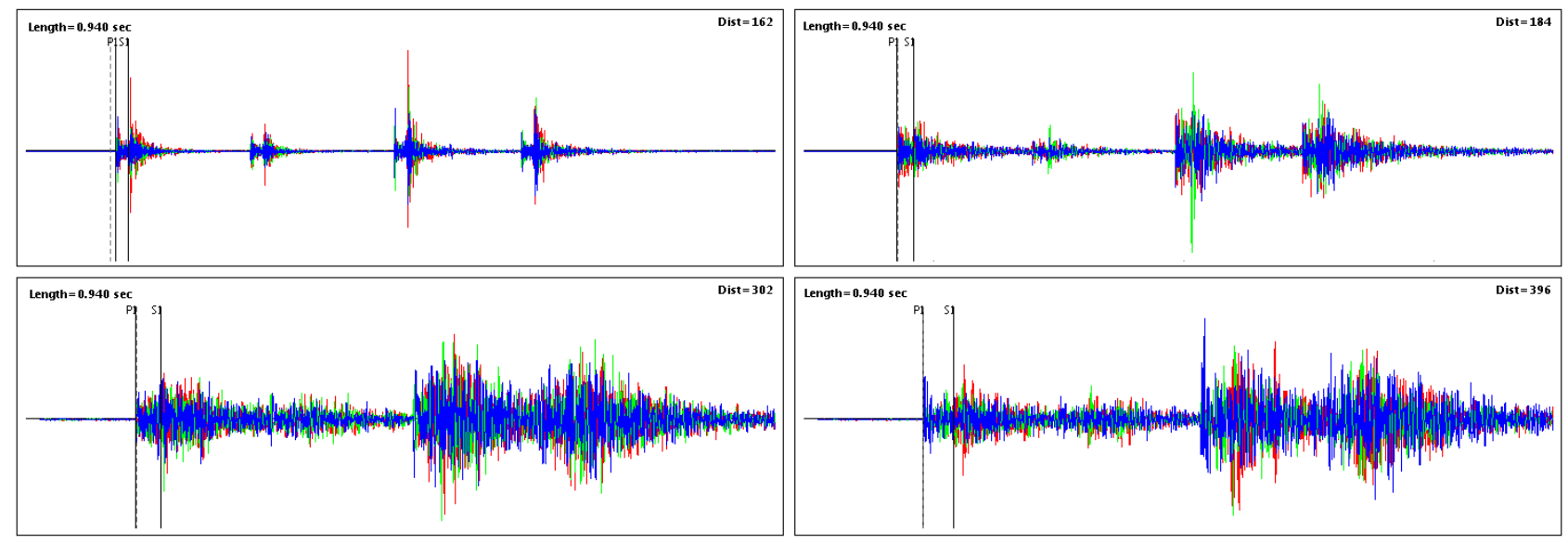

Figure 2 Typical seismograms of a series shot firing. Repetitive seismic signals have a resembling pattern - time delay between arrivals of $\mathrm{P}$ - and $\mathrm{S}$-waves, polarisation of ground motion. This indicates the proximity of their sources and the similarity of the source mechanisms

The signals from individual firings in Figure 2 are within one time buffer. In other instances the seismograms may contain seismic signals of only a single fired shot. This complicates visual classification of the event. 
The repetition effect is quantified by calculating the correlation of seismic waveforms for the analysed event with preceding and subsequent waveforms. A time window between 10 to 30 seconds is generally used to select the neighbouring waveforms. Seismic signals both in the same buffer (see Figure 2) as well as in the neighbouring events are considered in the calculation. A median value of the five highest stacked cross-correlation amplitudes is computed and the logarithm of this value is considered as the feature 'correlation of the waveforms'.

In general the feature varies between -2 and 0 . Values close to 0 usually indicate that multiple similar signals are in the proximity of the analysed event, whereas values lower than -1.0 implies either the absence of neighbouring signals or poor correlation with the considered event.

\section{2. 3 High-frequency versus low-frequency radiation}

Blasts or explosions usually radiate higher frequency waves compared to normal seismic events (Bormann, 2002). Various combinations of seismic parameters, such as the ratio between seismic moment and corner frequency, apparent stress, energy index (Mendecki, 1997), etc., may be used to capture a difference in the frequency content of seismic events.

An apparent stress $\sigma_{A}$, which is proportional to the ratio of seismic energy to seismic moment $\left(\sigma_{A} \propto E_{S} / M\right)$, is preferred since the evaluation of seismic energy is more robust than corner frequency. The energy index is not a preferable choice as it requires manual fitting of the $E_{S}$ versus $M$ ratio.

The logarithm of apparent stress is considered as the feature 'high versus low frequency radiation'. Blasts generally have higher $\log \left(\sigma_{A}\right)$ than normal events.

\section{2. 4 Radiation pattern}

There is a fundamental difference in radiation of seismic waves between blasts and normal events. A blast radiates predominantly P-waves that have positive polarity (first motion directed outward from the source) for all seismic sensors. Shear fracturing or slip on a surface in a homogeneous medium radiates $S$-waves that are stronger than P-waves. The polarity of P- and S-waves depends on the direction from the source (Figure 3).

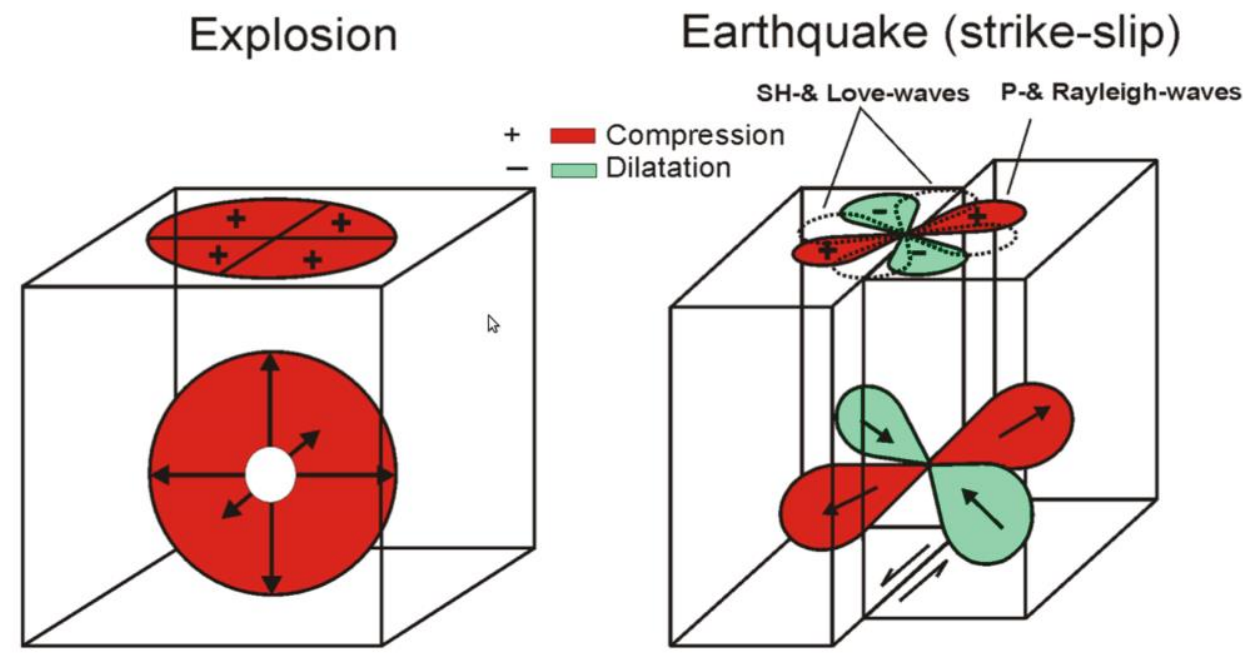

Figure 3 Radiation pattern of an idealised explosion and of a strike-slip earthquake along a vertically dipping fault (Bormann, 2002). The arrows show the directions of compressional (outward, polarity + , red shaded) and dilatational (inward, polarity, green shaded) motions in P-wave 
The basic characteristics of radiation can be described by a seismic moment tensor. This tensor represents a set of fictitious dipoles acting on a point in the source area. The moment tensor makes it possible to describe the low-frequency amplitudes and polarities of seismic waves. Inverting the moment tensor from the observed waveforms and analysis of its components allows the discrimination of blasts from slip-type and, even, from crush-type events.

The standard processing practice usually does not include the evaluation of the source mechanism for each recorded event. Thus making use of the discriminating feature based on a source mechanism is not feasible and therefore the current analysis restricts the radiation pattern signature to the ratio of amplitudes of P- and S-waves. Polarities of the waves and spatial distribution of the sites are ignored. This enhances the stability of the feature, but reduces the resolving power.

A generalised relation between amplitudes of $\mathrm{P}$ - and $\mathrm{S}$-waves is provided by comparing $M_{P}$ (seismic moment estimated from P-waves) with $M_{S}$ (seismic moment estimated from S-waves). The routine procedure of source parameter calculation in widely used processing software is based on a shear crack model, imposing a corresponding radiation pattern correction to P- and S-waves. Thus for the normal events it is expected that $M_{P} \approx M_{S}$, while for other source processes the applied radiation pattern correction is not valid, and consequently, $M_{P} \neq M_{S}$.

The logarithm of the ratio of $M_{P}$ to $M_{S}$ was selected as the feature 'radiation pattern', where $\log \left(M_{P} / M_{S}\right) \approx 0$ for normal events and $\log \left(M_{P} / M_{S}\right)>0$ for blasts.

\section{Calibration}

Calibrating the blast discriminator for a specific mine requires the compilation of representative sets of normal events and blasts.

In some cases this can be done by means of visual waveform inspection (repetition of seismic signals, ratio of amplitudes of P-and S-waves, etc.) as well as comparing the times and source locations with blast data provided by the mine. When the visual inspection is not conclusive a more sophisticated approach, involving the analysis of the source mechanisms, has to be used. Moment tensor inversion, which accounts for the ambiguities, makes it possible to classify the seismic event (Ford et al., 2010) and assign it to an appropriate calibration dataset (blasts or normal events).

As soon as the calibration datasets are compiled, the mean values $\boldsymbol{\mu}_{\mathbf{x}}$ and $\boldsymbol{\mu}_{\mathbf{Q}}$, as well as covariance matrices $\boldsymbol{\Sigma}_{\mathbf{X}}$ and $\boldsymbol{\Sigma}_{\mathbf{Q}}$ of the discriminating features are calculated.

The details of the calibration procedure for four Australian mines are presented in Figure 4. Histograms are used to illustrate the behaviour of the discriminating features, whereas lines are used to display the feature approximations by a multivariate Gaussian distribution.

Figure 4 shows that the best performing discriminating feature for the considered mines is 'correlation of the waveforms'. It provides the best separation between the populations of blasts and normal events. 
Mine A
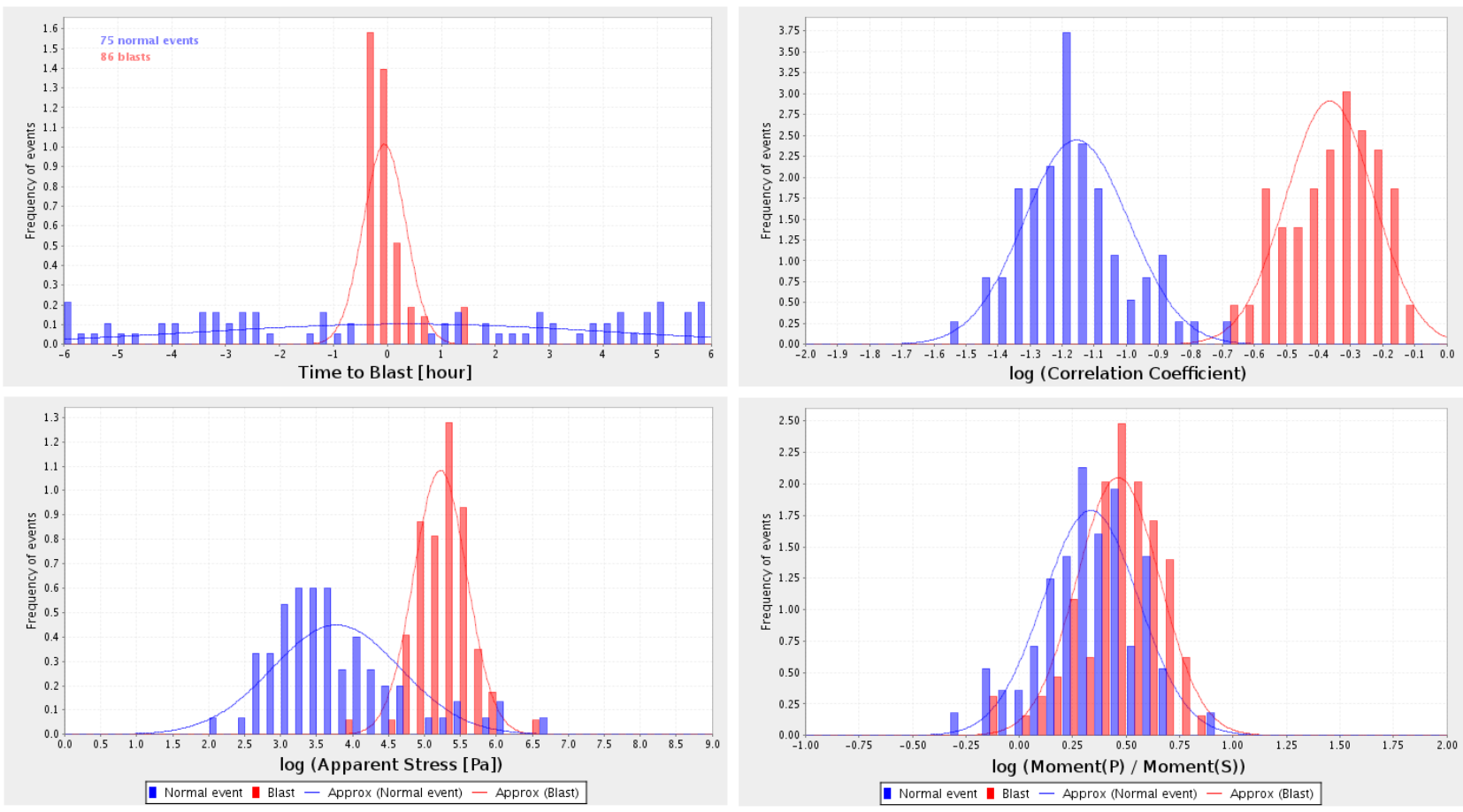

Mine B
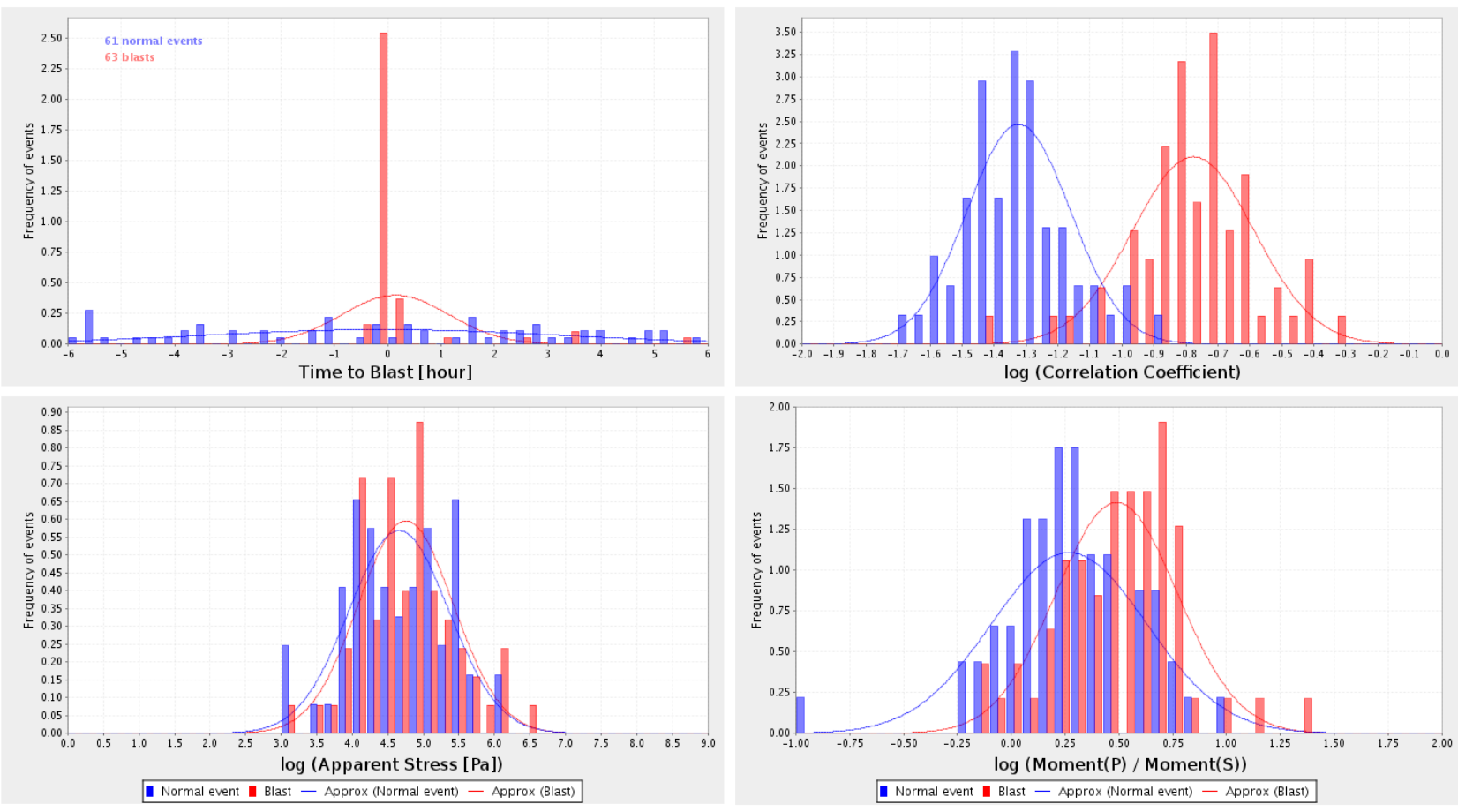

Figure 4 Distributions of the measured features for the calibration datasets of two mines (A and B) are displayed as histograms. Multivariate Gaussian approximations of these are shown using solid lines. The 'correlation of the waveforms' (top right graph for each mine) is the best performing discriminating feature as it provides maximum separation between normal events and blasts 
Mine C
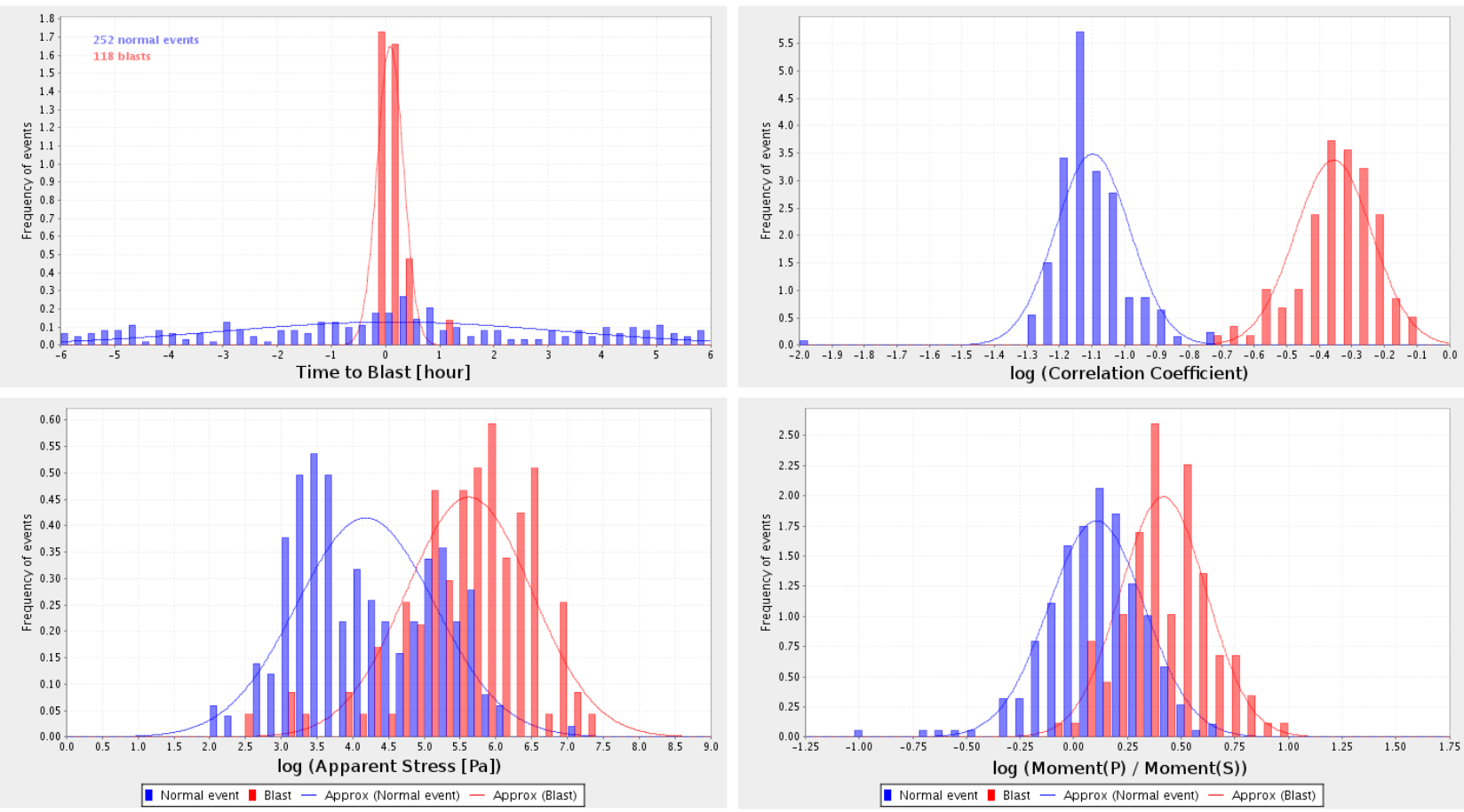

Mine D
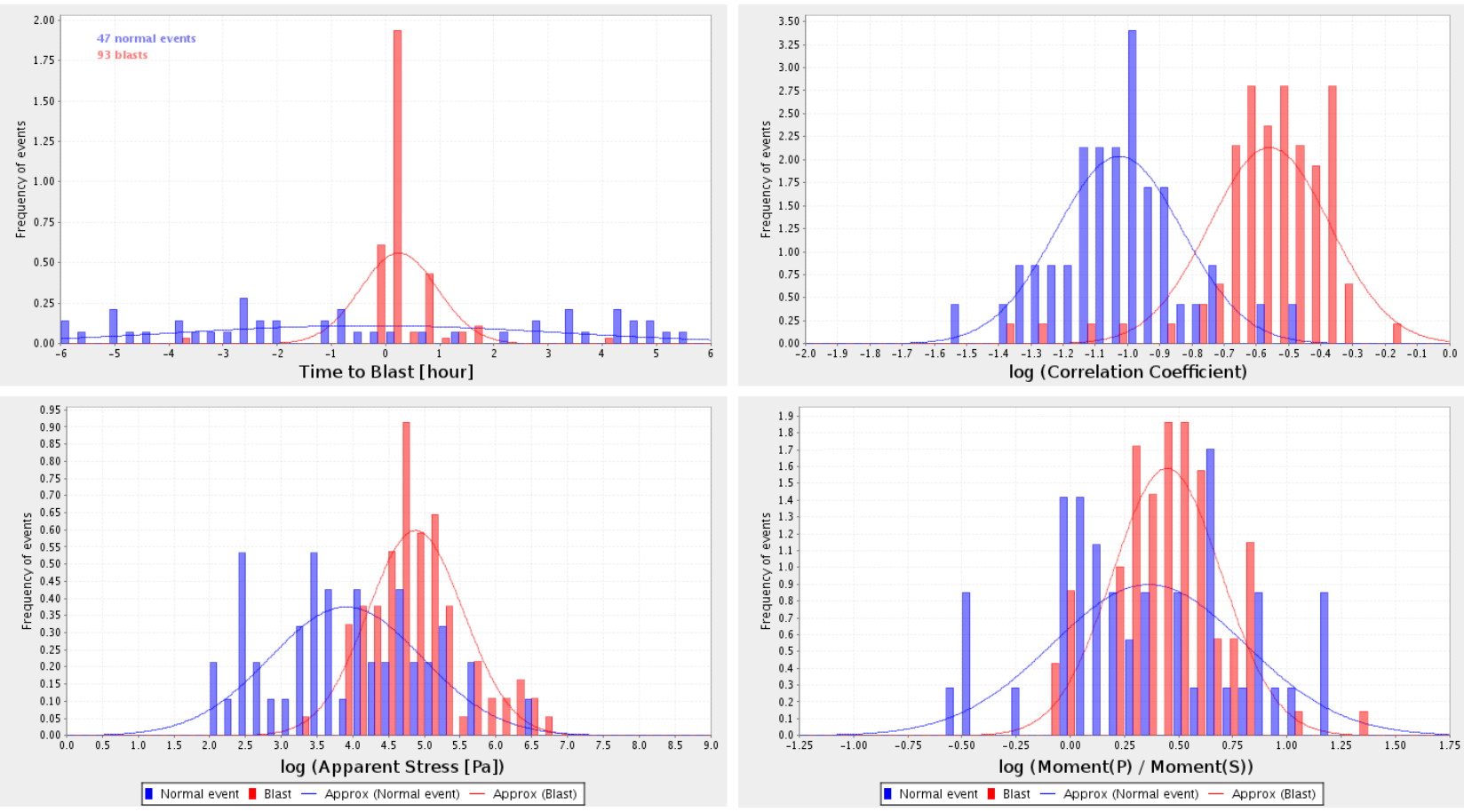

Figure 4 (continued) Distribution of the features for the calibration datasets of two mines (C and D). The 'time of day' feature (top left graph for each mine) constraints the population of blasts. The features of 'high versus low frequency radiation' (bottom left graph) is efficient for the mines $A$ and $C$, but not efficient for the mine B. The feature 'radiation pattern' (bottom right graph) provides a slight separation between blasts and normal events 
The 'time of day' feature ('time to blast' in Figure 4) constrains the population of blasts. The dispersion of the Gaussian approximation is relatively small. Such a constraint generally does not exist for normal events, as they may occur both during and outside the blasting time. As a result the Gaussian approximation has large dispersion.

The feature 'high versus low frequency radiation' ('log (Apparent Stress [Pa])') shows a good separation for only two of the mines ( $A$ and $C$ in Figure 4). From Figure 4 it is also visible that normal events have average values of apparent stress of approximately $1-30 \mathrm{kPa}$, whereas blasts have values of approximately $30 \mathrm{kPa}$ to $1 \mathrm{MPa}$.

The feature 'radiation pattern' does not provide a clear separation for the considered mines. A slight distinction of the log (Moment (P)/Moment(S)) exists between the two types of events. As expected, blasts show $M_{P}$ higher than $M_{S}$. However, the majority of the normal events also show $M_{P}>M_{S}$. This indicates a presence of non-slip-type events among the normal events.

Note that Figure 4 only visualises certain elements of the Gaussian approximation, such as the mean values $\left(\boldsymbol{\mu}_{\mathbf{X}}\right.$ and $\boldsymbol{\mu}_{\mathbf{Q}}$ ) and variances (diagonal components of the covariance matrices $\boldsymbol{\Sigma}_{\mathbf{X}}$ and $\boldsymbol{\Sigma}_{\mathbf{Q}}$ ). The correlations between individual features (non-diagonal components of the covariance matrices $\boldsymbol{\Sigma}_{\mathbf{X}}$ and $\Sigma_{\mathbf{Q}}$ ), also play an important role in the discrimination (Anderson and Taylor, 2002). These values are calculated during the calibration phase, but are not illustrated here.

Distributions of the discrimination function $G(\mathbf{v})$ calculated for the calibration datasets are presented in Figure 5.
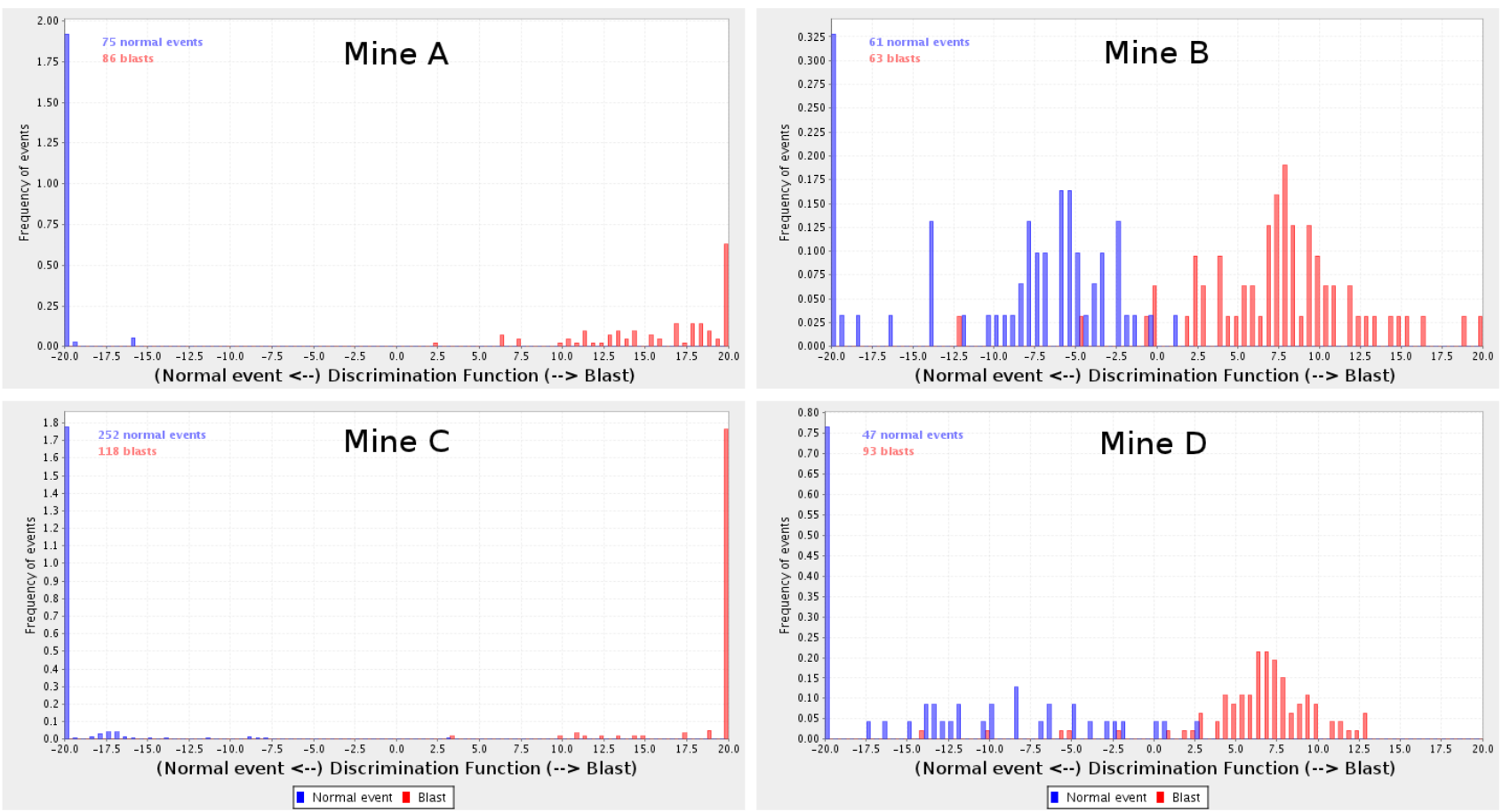

Figure 5 The values of the discrimination function $G(\mathbf{v})$ (Equation 1) for the calibration datasets. Values lower than -20 and higher than 20 are accumulated in the first and the last bars, respectively. The discriminator performs well for mines $A$ and $C$ since the values of the discrimination function for populations of blasts and normal events are separated. For mines $B$ and $D$, the discriminator is less accurate since some of the events, even for the calibration datasets. are misclassified, e.g. several blasts have $G(\mathbf{v})<0$, while some normal events have $G(\mathbf{v})>0$ 
The discriminator provides good separations between the blasts and normal events for mines $A$ and $C$, but less accurate separations for mines B and D.

\section{$4 \quad$ Application}

\section{1 Example 1}

A seismic event recorded in mine D (Figure 6) was chosen to demonstrate the mechanics of the discriminator. Since the event occurred during a blasting time and some waveforms show stronger P-waves, this event may be classified as a blast. However, the absence of a 'multi-shot' repetition pattern for all sites, as well as the presence of stronger S-waves for some sites, could be considered as attributes of a normal event. The calibrated discriminator can resolve such an ambiguity.

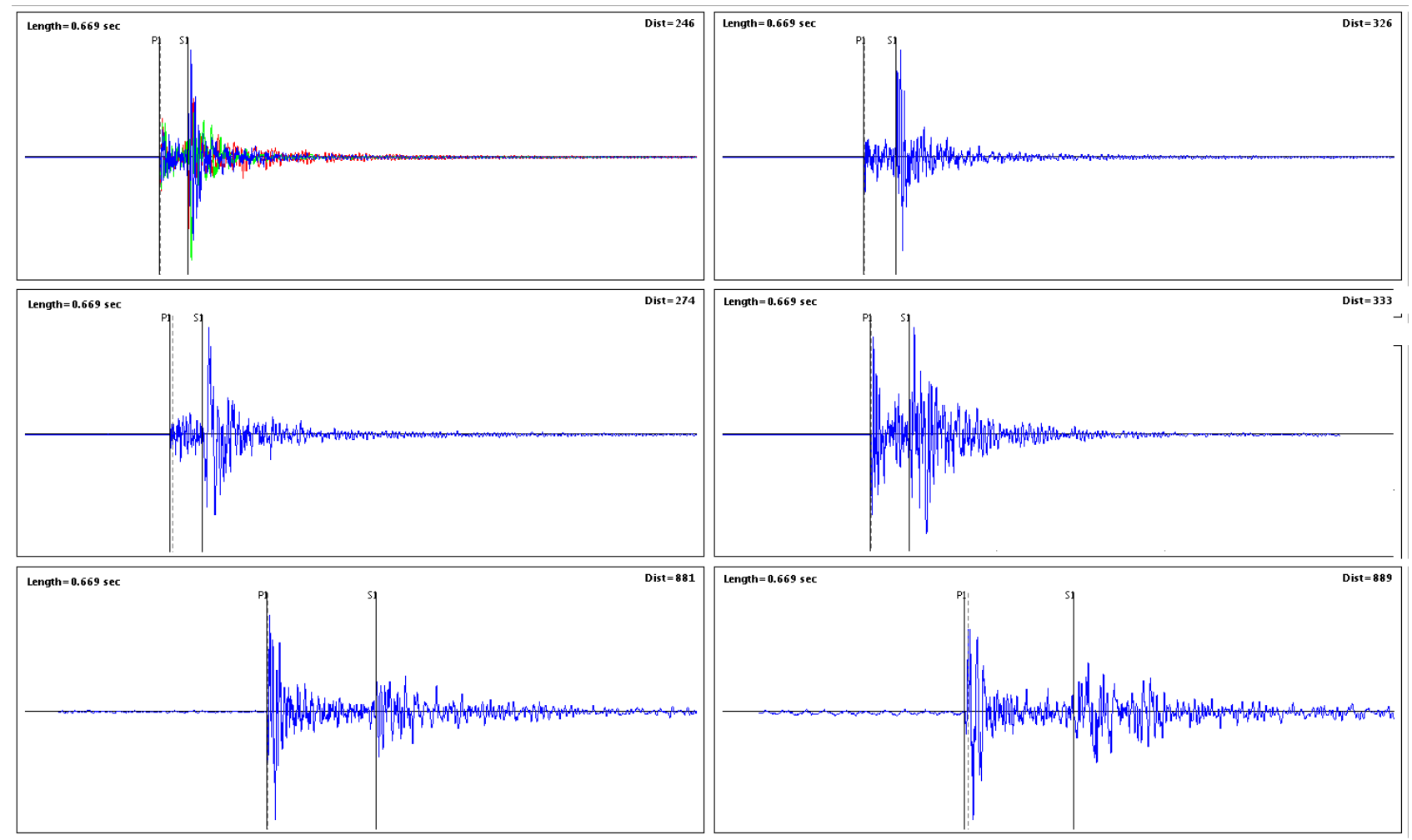

Figure 6 Seismic event recorded in mine D during blasting time. Visual features of the waveforms (absence of repetitive signals, strong $S$-wave at some sites and $\mathrm{P}$-wave at other sites) make it difficult to classify the event either as normal or a blast

The discriminating features for the event appear to be closer to the population of blasts (Figure 7). The value of the discrimination function $G(\mathbf{v})$ is 11 , strongly indicating that the event is a blast. 

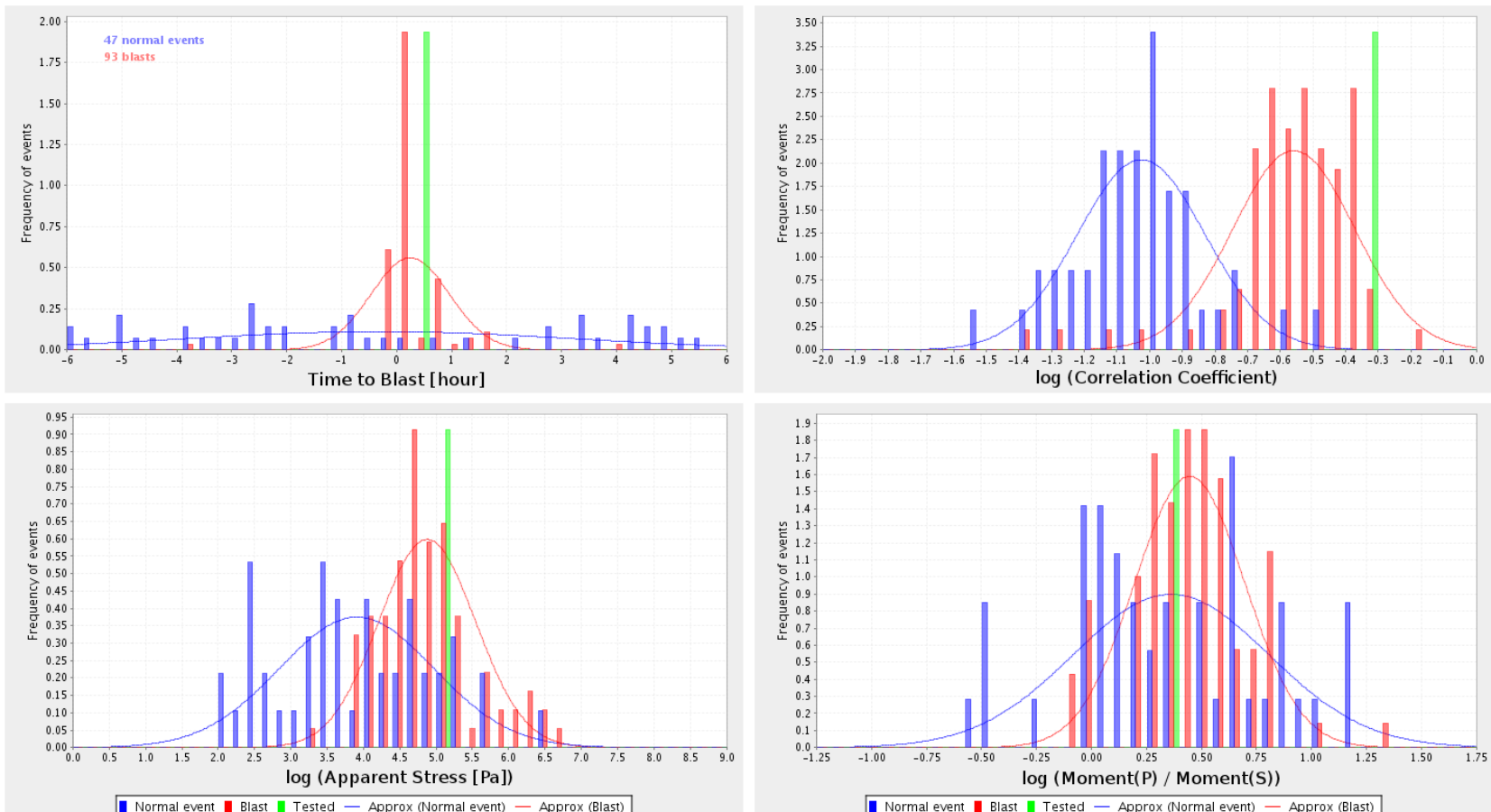

Figure 7 Details of the discrimination of the event (shown in Figure 6). Individual features for this event (green bars) are compared with the features of the calibration datasets (red bars for blasts and blue bars for normal events). The value of the discrimination function is positive $(G(\mathbf{v})=11)$, indicating that the event is a blast

\section{2 Example 2}

This example demonstrates the results of a large-scale application of the discriminator.

The calibrated discriminator was applied to 7,053 events that were recorded in mine B and manually classified as normal. After the discrimination 1,431 of the events $(\sim 20 \%)$ were reclassified as blasts. Note from Table 1 that the ratio of discriminated blasts and normal events is not uniformly distributed, but varies for different magnitude ranges. It was found that original database contained a significant amount of blasts for the magnitude range $-0.5<\mathrm{m}<0.5$.

Table 1 Results of application of the discriminator to the seismic data of the mine $B$

\begin{tabular}{cccc}
\hline Magnitude Range & Original Database & Discriminated Normal Events & Discriminated Blasts \\
\hline$m>-2.0$ & $4,434(100 \%)$ & $3,615(82 \%)$ & $819(18 \%)$ \\
$m>-1.0$ & $715(100 \%)$ & $403(56 \%)$ & $312(44 \%)$ \\
$m>0.0$ & $180(100 \%)$ & $61(34 \%)$ & $119(66 \%)$ \\
$m>1.0$ & $9(100 \%)$ & $9(100 \%)$ & $0(0 \%)$ \\
\hline
\end{tabular}

The application of the discriminator affected the statistical characteristics of the seismic data. The power law magnitude distribution provides a better fit to the discriminated dataset for magnitudes greater than -1.5 , as compared to the original dataset (Figure 8). 

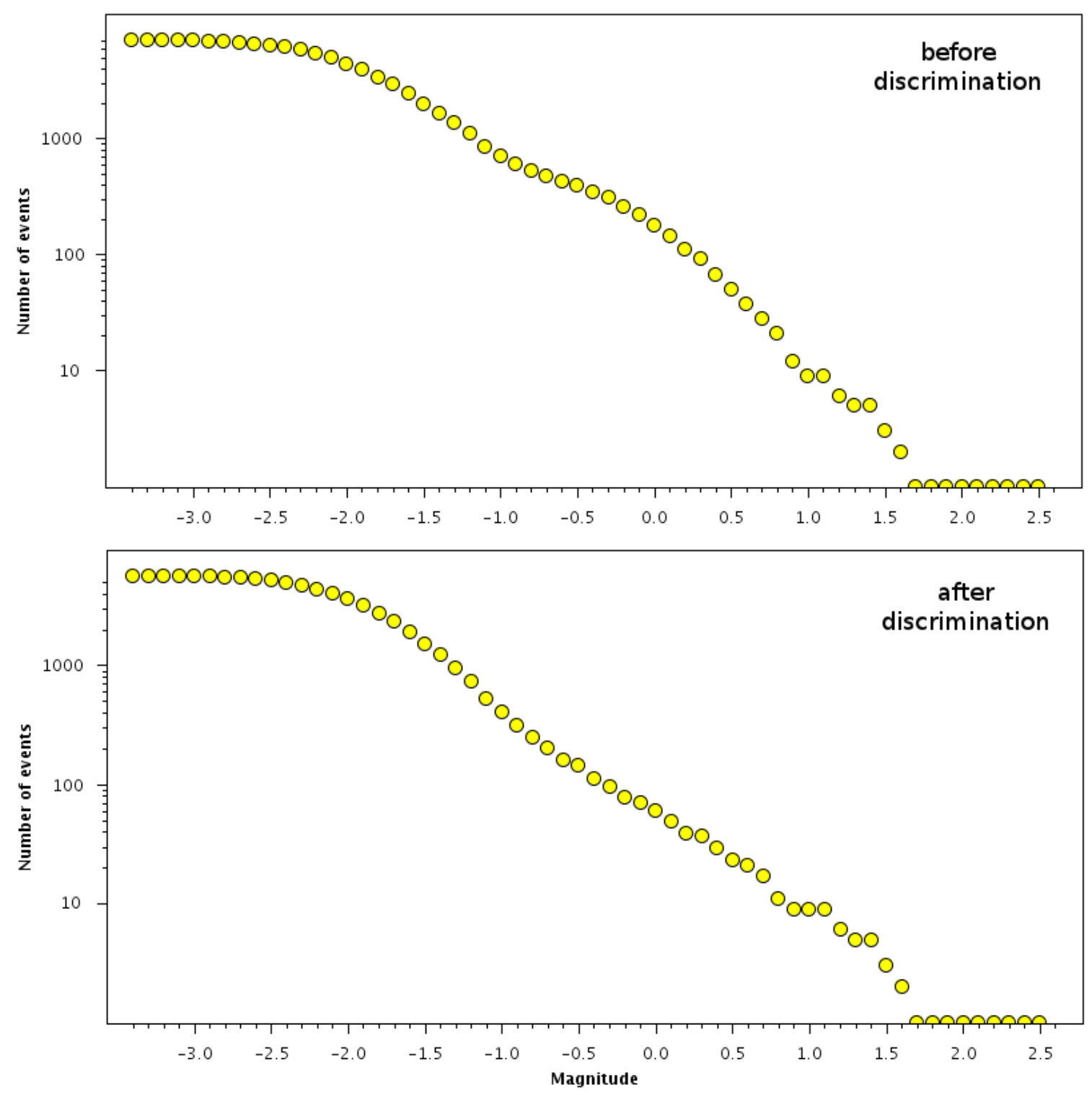

Figure 8 Magnitude distributions of seismic events recorded during a four month period in mine B. The top plot is based on the original seismic dataset of 7,053 events that were manually classified as normal. The bottom plot shows the same dataset after the discriminator eliminated the blasts that were incorrectly classified as normal events. Note a hump for the magnitude range $-0.5<\mathrm{m}<0.0$ in the original distribution. This is not observed for the improved dataset

\section{Conclusions}

The multivariate maximum-likelihood Gaussian classifier technique was implemented to provide discrimination between normal events and blasts in mines. Four independent features of seismic signals and source parameters were selected as components of the discriminator:

- time of occurrence

- similarity of the seismic signals with the neighbouring waveforms

- ratio of high-frequency and low-frequency radiation

- radiation pattern. 
The technique was calibrated and applied routinely to a number of mines for which Institute of Mine Seismology provides seismological services. Application of the discriminator changed the processing concept. Currently, every seismic event is either rejected or processed by a human processor. The processed event is then classified by the discriminator. This brings consistency to the seismic catalogues in terms of the source types. An additional benefit is that blasts are also processed and thus can be used to monitor the quality of the processing, i.e. the seismic location of blasts should not be far from their actual position. Source parameters of the blasts can also provide additional information about the state of the rock mass.

It is important to note that the output of the discriminator depends on the datasets of blasts and normal events that are used for the calibration. Properties of seismicity, as well as the blasting procedures (blasting time, firing technique) may change over time which could lead to discrimination results becoming less accurate. In this case the discriminator has to be recalibrated. Cooperation between geotechnical engineers and mine seismologists is important to maintain the representative calibration datasets.

\section{Acknowl edgements}

The author thanks the mines that permitted the presentation of their seismic data. Special thanks to Gys Basson from Institute of Mine Seismology for his input to the manuscript.

\section{References}

Anderson, D.N. and Taylor, S.R. (2002) Application of regularized discrimination analysis to regional seismic event identification, Bulletin of the Seismological Society America, Vol. 92, pp. 2391-2399.

Booker, A. and Mitronovas, W. (1964) An application of statistical discrimination to classify seismic events, Bulletin of the Seismological Society America, Vol. 54, pp. 961-971.

Bormann, P. (2002) New Manual of Seismological Observatory Practice, GFZ German Research Centre for Geosciences, Potsdam, version 2002 in two volumes, $1250 \mathrm{p}$.

Brady, B.H.G. and Brown, E.T. (2005) Rock mechanics for underground mining, Kluwer Academic Publishers, 628 p.

Ford, S.R., Dreger, D.S. and Walter, W.R. (2010) Network Sensitivity Solutions for regional moment-tensor inversions, Bulletin of the Seismological Society America, Vol. 100, pp. 1962-1970.

Mendecki, A. (1997) Seismic monitoring in mines, Chapman and Hall, 262 p.

Taylor, S.R. (1996) Analysis of high-frequency Pg/Lg ratios from NTS explosions and Western U.S. earthquakes, Bulletin of the Seismological Society America, Vol. 86, pp. 1042-1053. 